ECIPERÚ

\title{
Implementación de un Sistema de Seguimiento Automático de Satélites Pequeños para la recepción e Interpretación de Señales Beacon desde la Estación Terrena del CTIC-UNI
}

\section{Implementation of an Small-Satellites Automatic Tracking System to Reception and Interpretation of Beacon Signals from CTIC-UNI's Ground Station}

\author{
Dante Inga, Iván Velásquez y Dane Cachi
}

Centro de Tecnologías de Información y Comunicaciones - CTIC UNI, Universidad Nacional de Ingeniería (UNI), Av. Túpac Amaru 210 - Rímac / Lima 25 - Perú.

\section{RESUMEN}

En las últimas décadas las universidades e institutos tecnológicos se han sumado a la construcción de satélites artificiales, desarrollando satélites pequeños con fines de investigación en el campo aeroespacial. Cada uno de estos satélites necesita por lo menos una estación en tierra, que usa hardware y software, para comunicarse con el satélite. Además, las estaciones terrenas disponen de sistemas informáticos para gestionar los datos obtenidos del satélite. El presente documento describe los procedimientos para realizar el seguimiento automático de satélites pequeños con el fin de recibir señales Beacon, es decir, información básica que envían los satélites pequeños de manera periódica en forma de señales de radio-frecuencia. El sistema hace uso de un conjunto de equipos hardware: radio, antena, rotor, TNC, los cuales tienen por función recibir la información del satélite; el sistema también hace uso de software para la gestión remota de los equipos y de la información recibida. Los satélites artificiales pequeños son normalmente de órbita baja, es decir, orbitan alrededor de la tierra a una altura de entre 200 y $800 \mathrm{Km}$, trasladándose a grandes velocidades respecto de la estación terrena. Por ello, la posición del transmisor del satélite y la frecuencia de la señal recibida cambian continuamente, razón por la que se requiere un ajuste de la posición de la antena y de la frecuencia de la radio, lo que se hace muchas veces de manera manual. El control automático del cambio de la frecuencia de la Radio y la orientación de la antena mejoran la recepción de las señales Beacon emitidas por el satélite. Los valores de frecuencia y ángulos serán obtenidos por un software predictor de órbitas, y se manejarán los equipos gracias a librerías open-source; además utilizando las ventajas del internet, podemos controlar el sistema desde cualquier PC con acceso a internet. El objetivo principal es mejorar la recepción de señales satelitales, disminuir la cantidad de ruido, facilitar el control de la estación terrena y obtener una decodificación automática con pocos errores. La información de una señal Beacon se encuentra en código Morse, es por eso que es necesario hacer uso de un TNC para decodificar dicha información. El software para gestión de la información obtenida de satélites pequeños está basado en el proyecto Mercury, adaptado al hardware de la estación terrena de CTIC-UNI. Se consiguió la decodificación en línea de la señal Beacon de varios satélites pequeños que se encuentran en funcionamiento a la fecha.

Descriptores: satélite, seguimiento automático, Beacon, estación terrena, Internet

\section{ABSTRACT}

In recent decades, universities and technology institutes have joined the construction of artificial satellites; they are developing small satellites for research in aerospace. These satellites require at least one ground station, which uses hardware and software to establish communication with the satellite. In addition, ground stations have computerized systems to manage data from the satellite. This document describes the procedures for automatic tracking of small satellites to receive Beacon signals, i.e. basic information that are sent by small satellites on a regular basis in the form of radio-frequency signals. The system uses a set of hardware devices such as radio, antenna, rotor, TNC, which will be responsible for receiving satellite data, the system also uses 
software for remote management of equipment and data received. Small satellites are usually low orbit, i.e., they are orbiting around the earth at an altitude of between $200 \mathrm{Km}$ and $800 \mathrm{Km}$, moving at high speeds relative to ground station. Therefore, the position of the satellite transmitter and the frequency of the received signal changes continuously, then antenna orientation and radio frequency adjustment are required, these often are done manually. Automatic control of radio frequency and antenna orientation improves the reception of Beacon signals emitted by the satellite. The frequency shift and antenna angle values are obtained by orbit predictor software and equipment will be handled by open-source libraries; also, using the advantages of the internet, we can control the system from any PC with Internet access. The main objective is to improve the reception of satellite signals, reduce noise level, facilitate earth station monitoring and get automatic decoding with few errors. The information obtained through Beacon signal is coded using Morse code, which is why it is necessary to use a TNC to decode the information. The management software for small satellite data obtained is based on Mercury project, adapted to the hardware of CTIC-UNI ground station. We achieved on-line decoding of Beacon signals from several small satellites that are operational to date.

Keywords: satellite, automatic tracking, Beacon, ground station, Internet

\section{INTRODUCCIÓN}

El diseño y construcción de satélites artificiales ya no es sólo para grandes organizaciones estatales o comerciales, sino que se realiza también por radioaficionados desde el lanzamiento del satélite OSCAR [1] en 1961, y por universidades e institutos tecnológicos posteriormente, para participar en experimentos de propagación de ondas de radio y tomar datos del espacio cercano a la Tierra. Estos satélites, que suelen ser de tamaño pequeño, están formados básicamente por una estructura metálica, un módulo de energía, un módulo de control, un sistema de comunicaciones y una carga útil que puede ser una cámara o algún otro sensor.

El sistema de comunicaciones del satélite artificial se encarga de enviar los datos adquiridos hacia la Tierra por medio de ondas electromagnéticas de radiofrecuencia; por tanto, se implementa un sistema análogo en tierra para recibir la información y enviar comandos al satélite, el cual se conoce como estación terrena, como se explica en [2]. Sin embargo, se toman en cuenta ciertas consideraciones para la recepción de datos provenientes del satélite, como la variación en la dirección y frecuencia de las ondas de radio recibidas, debido a la gran velocidad con la que se desplaza el satélite respecto de la estación terrena.

Para los satélites pequeños existe software de libre uso como el Orbitron para Windows o el Gpredict para Línux, que permiten el control de los equipos de la estación terrena para un seguimiento automático del satélite. Sin embargo, estos programas no son lo suficientemente flexibles para la diversidad de equipos que existen, y requieren de usuarios avanzados para la modificación del software en los casos donde es posible.
Se plantea la implementación de un sistema que consiste en hardware y software, para el control de los equipos de una estación terrena y el manejo de la información enviada por satélites pequeños, con el fin de interpretar específicamente las señales Beacon enviadas por casi todos los satélites de este tipo. El usuario final puede acceder al sistema por Internet, iniciar el seguimiento y recolección de datos del satélite, y observar la información decodificada.

\section{MÉTODO EXPERIMENTAL}

Los equipos con los que se probó el sistema son:

-Antenas Xquad para VHF y UHF.

-Radio transceptor ICOM IC-910H.

-Rotor de antena Yaesu G-5500.

-Modem para CW (TNC).

-PC con procesador de 2 núcleos.

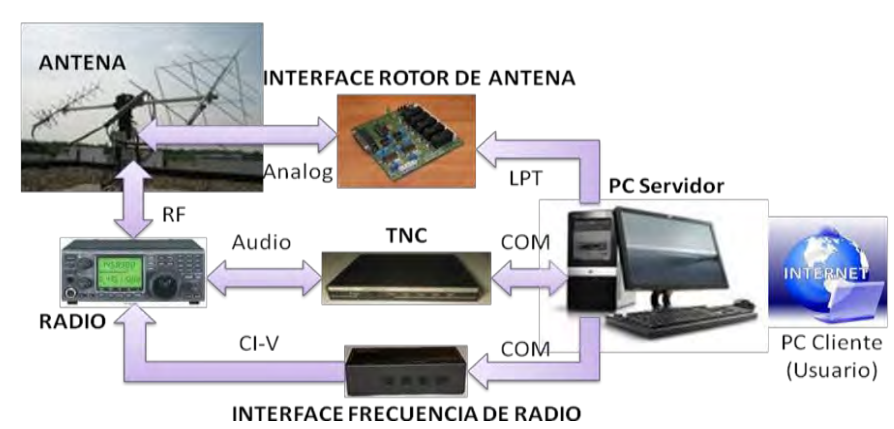

Figura 1: Equipos de una estación terrena.

Muchos de los componentes de la estación terrena son capaces de un control asistido por computadora. La antena de posicionamiento de motores puede ser dirigida a través de la tarjeta de expansión de la PC. La radio tiene una interfaz en serie para ajustar las frecuencias y determinar los niveles de recepción de la señal.

Para facilitar la automatización de los controles de la estación terrena de seguimiento de satélites se ha desarrollado el software Mercury por el Grupo de 
infraestructuras de software (SWIG) y el Laboratorio de desarrollo de sistemas del espacio (SSDL) de la Universidad de Stanford, esto se explica en [3].

El siguiente diagrama muestra el flujo de datos y comandos del Software Mercury

Data flow:

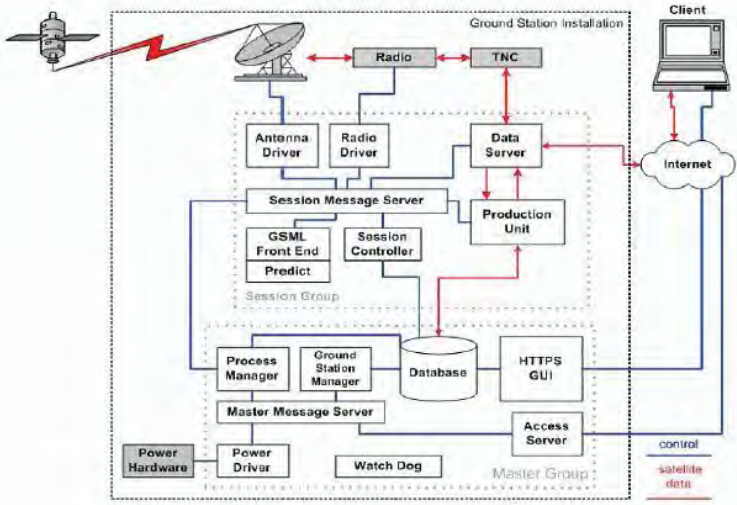

Figura 2: Diagrama de bloques del Software Mercury.

El Software Mercury tiene como funcionalidad un módulo que se encarga de iniciar una sesión, que significa el inicio del seguimiento del satélite, como se ve en la figura 3 , se selecciona el satélite, la hora de inicio de sesión y la hora fin de sesión, con esto podemos acotar el funcionamiento del seguimiento del satélite, a partir de ahí automáticamente el software está en funcionamiento y se recibe datos.

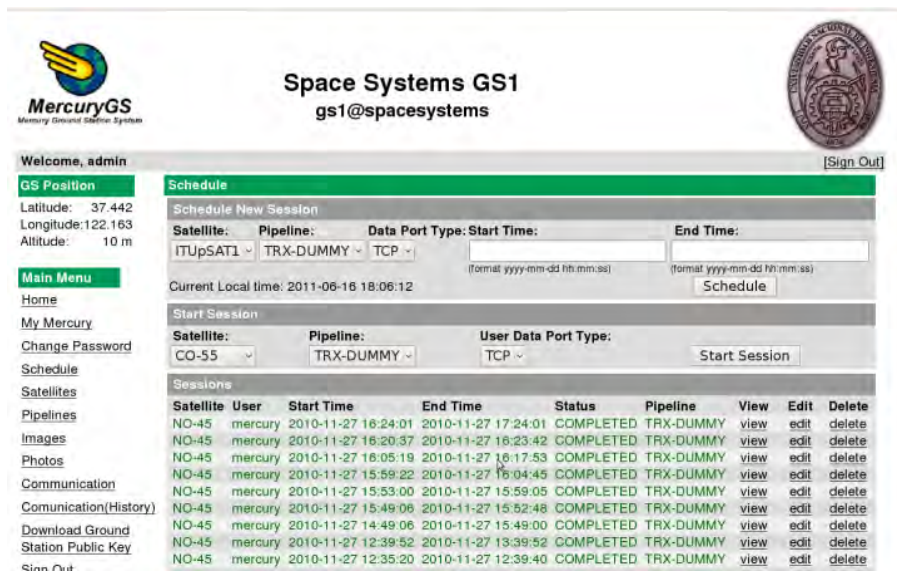

Figura 3: Módulo de programación de sesiones

Mientras el software se está ejecutando, los datos recibidos del satélite estarán mostrándose en una ventana, como se indica en la figura 4. Estos datos estarán disponibles a nivel local, pero también a nivel de la red, es decir podrán ser visualizados en línea desde cualquier punto de internet, para aquellos que estén interesados en ver los resultados del Beacon recibido.

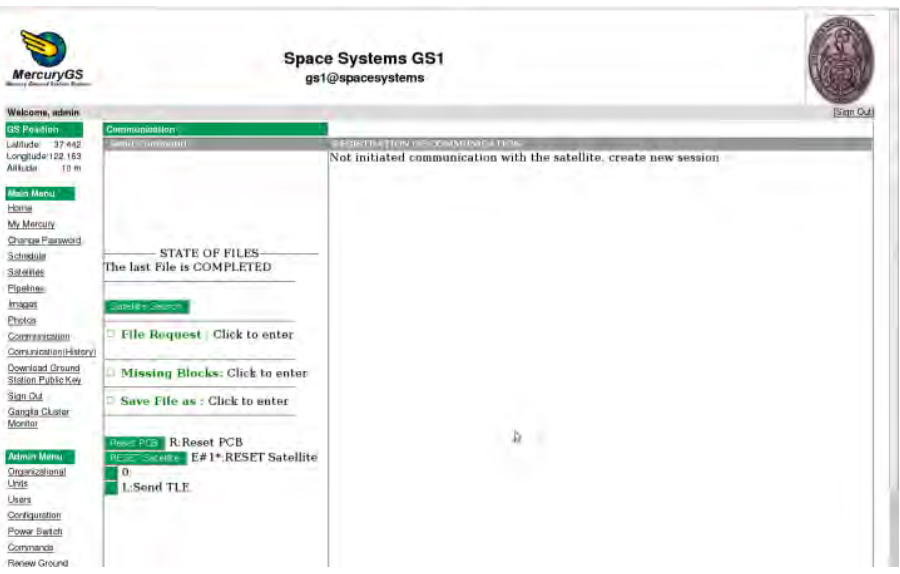

Figura 4: Módulo de recepción de datos

Uno de los beneficios del software usado en nuestro sistema es que la frecuencia nominal de la señal Beacon emitida por los satélites pequeños se puede establecer, por lo que se puede decidir la frecuencia de la señal de audio recibida por la radio. Una señal de Beacon es código Morse modulado en ASK; este tipo de modificación digital se explica en [4]. Si las frecuencias de las señales Beacon se ajustan a un valor estándar de $1200 \mathrm{~Hz}$ por ejemplo, se puede usar un demodulador sencillo, lo que significa una reducción de costo en el TNC.

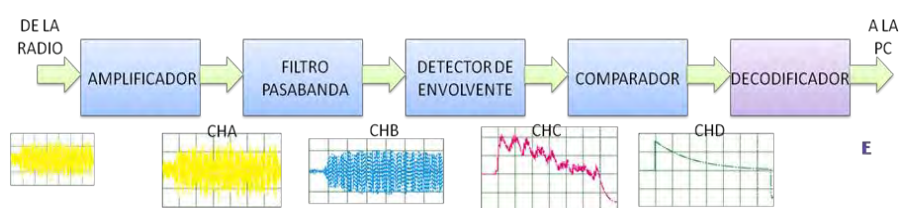

Figura 5: Diagrama de bloques del TNC usado.

La figura 5 muestra el diagrama de bloques del TNC usado para la decodificación de las señales Beacon emitidas por satélites pequeños. El amplificador es un amplificador operacional configurado como inversor, el filtro pasabanda es Butterworth de 4to orden implementado con amplificadores operacionales, el detector de envolvente es un diodo rectificador más un filtro RC pasabajo, el comparador también está basado en un opamp, y el decodificador es un microcontrolador PIC.

El modem recibe la señal Beacon audible del terminal de datos de la radio, la amplifica y luego la filtra para disminuir la cantidad de ruido. El detector de envolvente rectifica la señal y filtra los cambios rápidos, el comparador genera la señal digital y el decodificador identifica los cambios de nivel, y mediante el tiempo de duración de los pulsos puede saber si se trata de punto, raya, silencio de símbolo, 
o silencio de carácter. La figura 6 muestra el prototipo de implementación, a la izquierda el puerto serial para la PC y a la derecha el conector de audio para la radio.

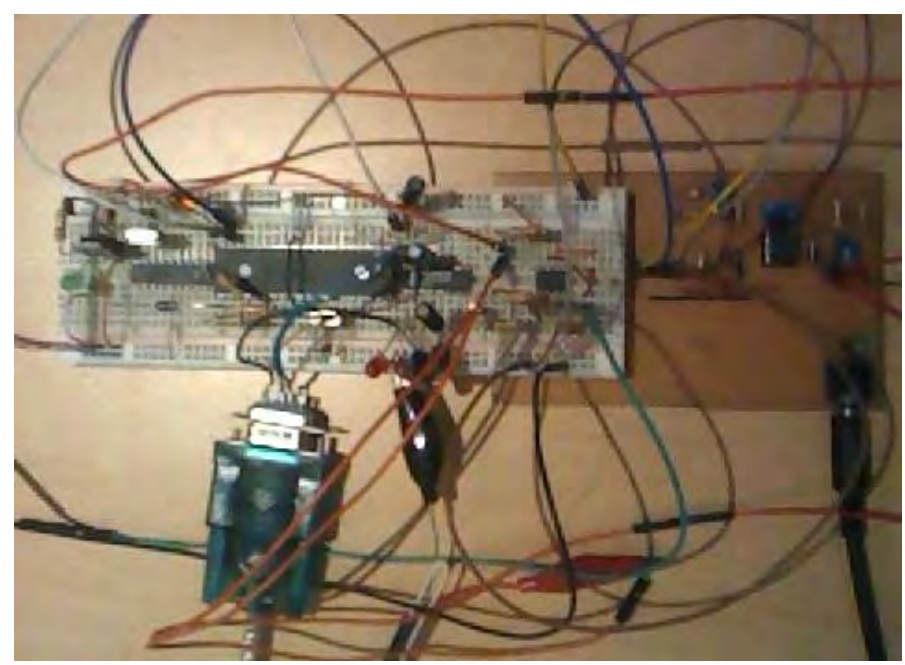

Figura 6: Prototipo de TNC para decodificar Beacon

\section{RESULTADOS Y DISCUSIÓN}

Se logró un buen ajuste de frecuencia recibida y decodificación de señales Beacon con un solo modem, hasta la fecha, para los satélites pequeños CO-55, RS-30, CO-57. Asimismo, el sistema se controla remotamente por Internet.

Las ventajas del sistema desarrollado respecto a los similares existentes son:

$>$ Calibración por software de los rotores de la antena, que permite corregir errores de orientación de la antena sin recurrir a cambios en el hardware.

$>$ Frecuencia nominal de señal Beacon ajustable, que permite estandarizar la frecuencia de audio recibida por la radio y usar un modem simple y más barato.

$>$ Los satélites nuevos se pueden agregar fácilmente al sistema, ingresando los parámetros orbitales y su modo de comunicación.

La figura 7 muestra la configuración del osciloscopio para la recepción de Beacon de 2 satélites pequeños y los colores representativos de cada canal.

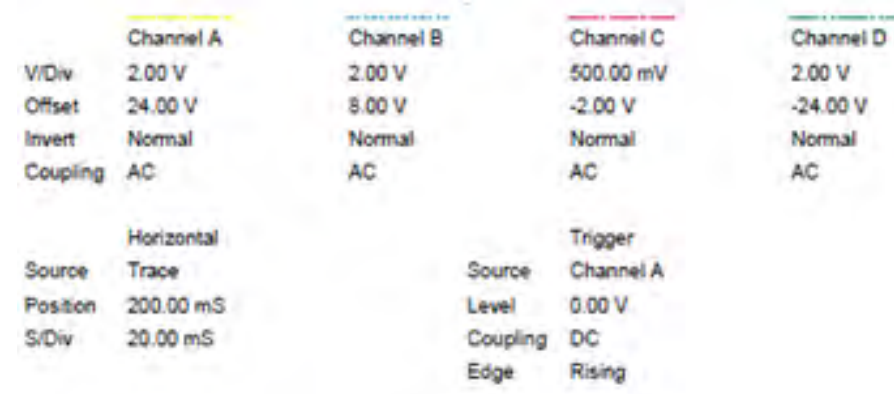

Figura 7: Configuración del osciloscopio

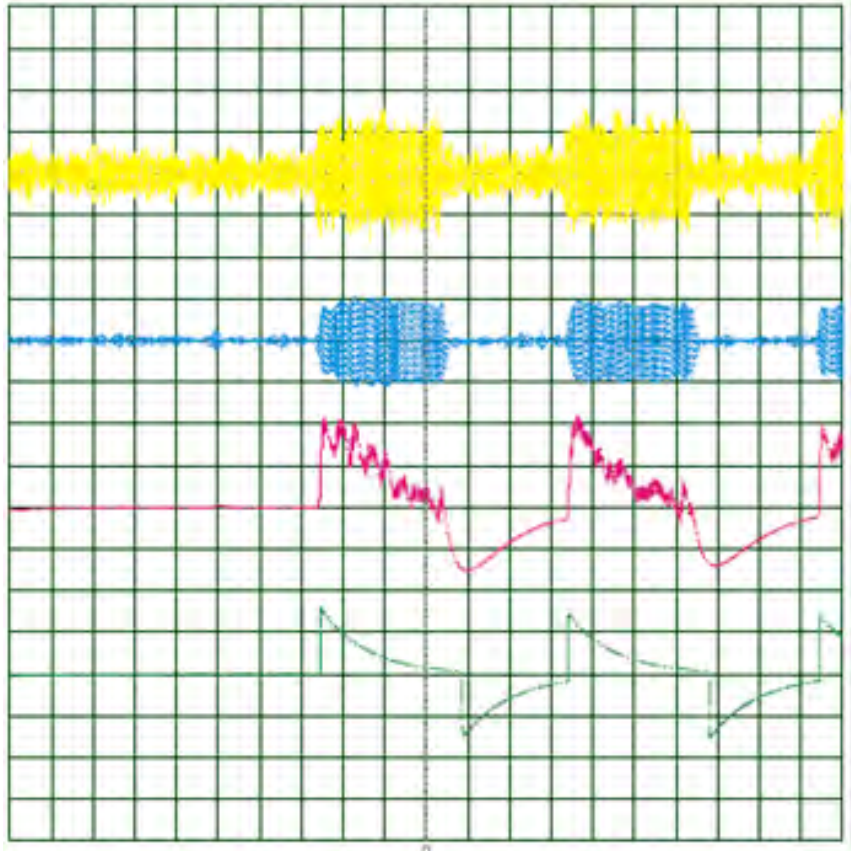

Figura 8: Gráficas para el satélite japonés CO-55

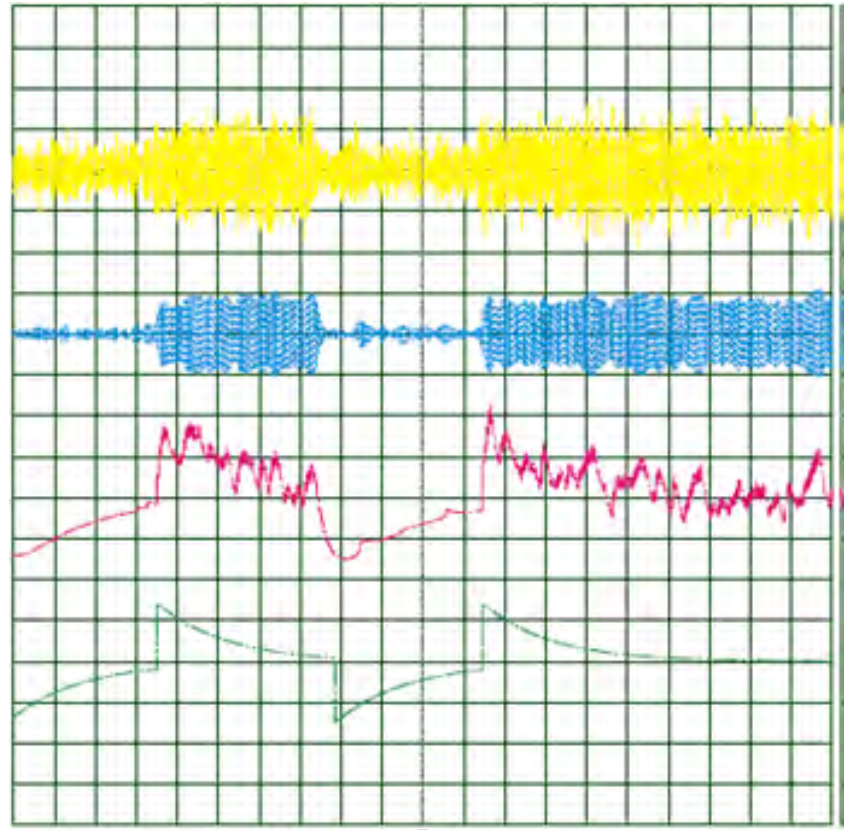

Figura 9: Gráficas para el satélite ruso RS-30

La figura 8 muestra la evolución de la señal Beacon captada por la radio y decodificada por el TNC, para 
el satélite CUTE1 (CO-55). La figura 9 muestra el procesamiento de las señales Beacon del satélite Yubileiny (RS-30).

En la tabla 1 se muestra una evaluación cualitativa de los sistemas existentes similares a nuestro sistema; esta forma de evaluación está basada en el estándar de calidad de software ISO 25010 [5]. EI criterio de evaluación consiste en los siguientes términos:

Bueno

Aceptable

Malo

Tabla 1: Cuadro de validación

\begin{tabular}{|l|l|l|l|}
\hline & Orbitron & Gpredict & Sistema usado \\
\hline Adaptabilidad & Bueno & Malo & Bueno \\
\hline Compatibilidad & Malo & Bueno & Bueno \\
\hline Usabilidad & Bueno & Bueno & Aceptable \\
\hline Confiablidad & Bueno & Aceptable & Aceptable \\
\hline Eficiencia & Malo & Malo & Bueno \\
\hline
\end{tabular}

\section{CONCLUSIONES}

En la actualidad se requiere que el software de seguimiento sea más flexible y adaptable a las necesidades modernas.

Para que la relación señal a ruido en recepción de Beacon sea buena, el satélite debe tener una elevación mayor a $30^{\circ}$.
La calidad de decodificación de señales Beacon mejora cuando la señal de la radio pasa por un filtro pasabanda angosto.

\section{AGRADECIMIENTOS}

El presente trabajo se pudo realizar gracias al Centro de Tecnologías de Información y Telecomunicaciones (CTIC-UNI).

\section{REFERENCIAS}

Todas las publicaciones citadas en el texto deberán ser presentadas en orden numérico de acuerdo a su aparición en el texto.

[1] K. Baker, D. Jansson, "Space Satellites from the World's Garage - The Story of AMSAT", National Aerospace and Electronics Conference, Dayton, Ohio, 23-27 May 1994.

[2] B. R. Elbert, "Introduction to Satellite Communication", 3th edition (2008).

[3] J. W. Cutler, C. A. Kitts, "Mercury: A Satellite Ground Station Control System".

[4] J. Proakis, "Digital Communication", 4th edition (2000).

[5] "International Standard ISO/IEC 25010", $1^{\text {st }}$ edition (2011)
E-mail: dingan@uni.pe ivelasqueza@uni.pe dcachi@uni.edu.pe

